\title{
Influence of hue, value, and chroma on the perceived heaviness of colors
}

\author{
KENNETH R. ALEXANDER and MICHAEL S. SHANSKY \\ Division of Visual Science, Illinois College of Optometry, Chicago, Ilinois 60616
}

\begin{abstract}
In addition to its information content, color is known to have certain affective qualities. We have investigated the assertion that colors have different apparent weights using a magnitude estimation technique. We find that the apparent heaviness of colors is an increasing function of chroma or saturation and a decreasing function of value or lightness. Hue has little influence on the apparent weight of color.
\end{abstract}

It has often been assumed that colors appear to have weight or heaviness. Thus assumption underlies many techniques in industry, art, and interior design. For example, color heaviness may affect task performance, since workers prefer to handle objects that appear lighter in weight (Birren, 1961). Artists have long utilized the color-weight principle in structuring the spatial relationships in their works (Pepper, 1949). Color arrangements are assumed to affect the esthetic balance of room designs (Trilling \& Nicholas, 1953).

Do colors appear to have weight, and, if so, which of the characteristics of color contribute to the perception of heaviness? Color has been classically defined in terms of both physical and psychological attributes. The physical attributes are wavelength, luminance, and colorimetric purity. If colors are viewed under reduced conditions in the absence of context effects, then the appearance of the colors can be described in terms of three psychological attributes-hue, brightness, and saturation. Each of these psychological attributes is primarily a function of a single physical attribute (e.g., hue changes primarily with wavelength). However, each psychological attribute is also affected by the other physical attributes, so that, for example, colors of a constant wavelength change in hue as luminance and colorimetric purity are varied.

If colors are viewed as surface colors under conditions of contrast, the brightness attribute becomes "lightness" and colors take on a white-gray-black appearance rather than being merely bright. One of the most common systems used to describe the appearance of surface colors is the Munsell renotation system, in which hue, lightness, and saturation are given the names hue, value, and chroma, respectively, and these color attributes are experimentally scaled. In the Munsell system, each color attribute is ordered in equal-sized steps, so that hue, value, and chroma form interval scales. ${ }^{1}$ Affective characteristics of color, such as weight, pleasantness, and warmth, could be expressed as a function of wavelength, luminance, and purity.
However, conventionally, such characteristics are more conveniently described in terms of hue, value, and chroma (Committee on Colorimetry, 1953).

The assumption that colors have apparent weight is based on early studies which generally found that "dark" colors appear heavier than "light" colors (Bullough, 1907; DeCamp, 1917; Monroe, 1926; Payne, 1958, 1961; Taylor, 1930; Warden \& Flynn, 1926). However, because of methodological shortcomings in these earlier studies, the relative contributions of hue, value, and chroma cannot be determined. For example, stimuli generally consisted of papers whose color attributes were ill defined and often confounded. Illumination levels and color temperature were not usually specified. The subjects' color vision was not assessed, and the data presented were often qualitative and introspective or were not analyzed statistically.

The color-weight hypothesis was recently reexamined using transilluminated stimuli rather than surface colors (Pinkerton \& Humphrey, 1974). ${ }^{2}$ It was concluded that the apparent weight of colors is determined by hue; brightness was found to have no effect on color weight. Unfortunately, this recent study also has methodological problems. First, the color attributes were not well controlled. Hue was not specified either in terms of dominant wavelength or in terms of hue as defined by the Munsell system. Instead, this attribute was simply called "colour," referring to the color name given to each filter. Brightnesses were equated by color matching, but since the stimuli were not surface colors, the effect of lightness on color weight was not determined (stimuli never appeared black or dark). In addition, since broad-band Wratten filters were used, neither colorimetric purity nor saturation was controlled. Finally, the subjects were apparently not screened for defective color vision.

In order to determine whether colors do appear to have weight, and to determine which of the psychological attributes of color contribute to the appearance of heaviness, we have reinvestigated the relationship between color and apparent weight using 


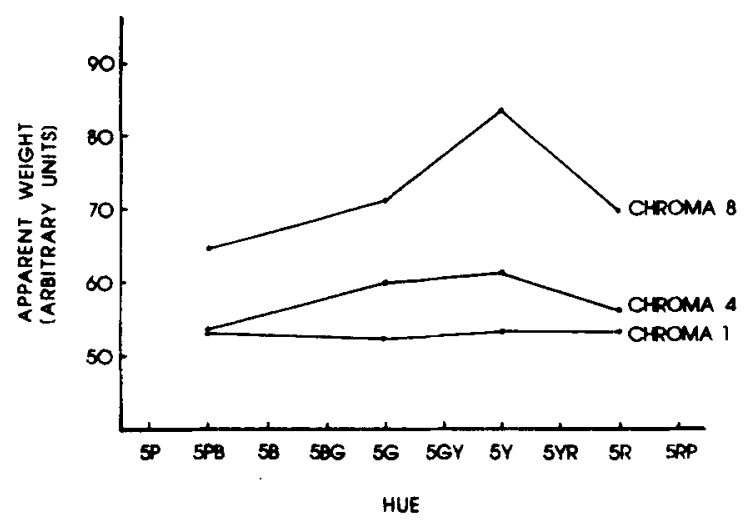

Figure 1. Apparent weight as a function of Munsell hue. Individual functions represent different chromas, as indicated. For all points, the standard errors (s.e.) are less than \pm 3 units.

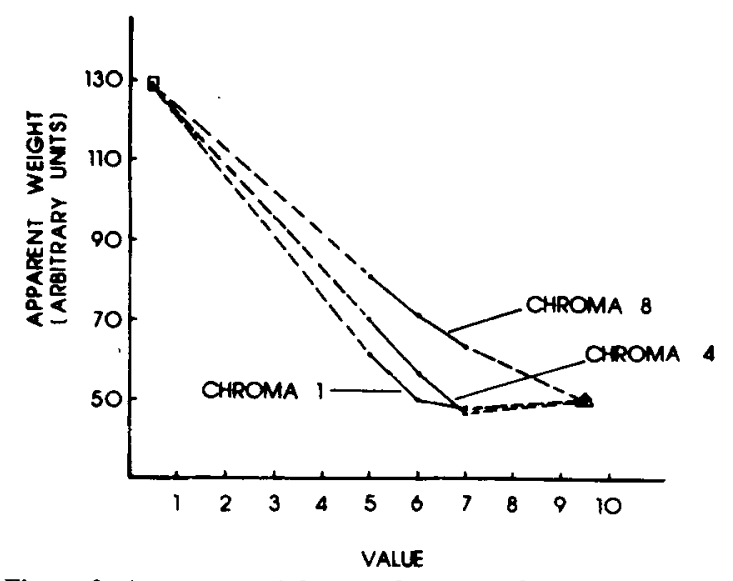

Figure 2. Apparent weight as a function of Munsell value. The triangle indicates the modulus (white), the square indicates black. The dashed lines are extrapolations. For all data points except black, the s.e. are less than \pm 3 units. For black, the s.e. $= \pm 8$ units.

stimuli matched as closely as possible for hue, value, and chroma. with colors chosen from the Munsell renotation system. ${ }^{3}$

\section{METHOD}

\section{Subjects}

Subjects were 10 male and 10 female optometry students, ages 20 to 25 . who were screened for normal color vision using the Ishihara (1971) plate test. None had yet had any formal instruction in the topic of color vision.

\section{Stimuli}

The subjects were tested individually in a black light-tight booth. They observed two squares. each of $3^{\circ} 15$ angular subtense. side by side on a dark gray background (approximately Munsell 2.5/) at an observation distance of $1 \mathrm{~m}$. with an intersquare separation (center to center) of $9^{\circ} 6^{\prime}$. The right square was white $(9.5 /)$; the left square was changed from trial to trial and was drawn randomly from a set of glossy $3 \times 5$ in. Munsell color chips, such that the set consisted of all combinations of four hues $(5 \mathrm{R}$-red. $5 \mathrm{Y}$-yellow. $5 \mathrm{G}$-green. SPB--blue), three values $(5.6 .7)$, and three chromas $(1,4.8)$ plus black $(0.5)$. The colors were chosen to satisfy two criteria: (1) that at least three different distinctive levels of each color attribute be chosen: (2) that there be no empty cells in the color matrix. ${ }^{4}$ The colors were illuminated by a Macbeth daylight lamp such that the luminances of the white square, black square, and background were 6.3 .0 .1 , and $0.5 \mathrm{fL}$, respectively.

\section{Procedure}

Data were accuired by magnitude estimation. Subjects were informed that the experiment was investigating the "heaviness" or "weight" of colors and were asked to assign numbers to the colored squares to represent the weight of each. The white square was arbitrarily assigned a modulus of 50 , and subjects were instructed to make ratio estimates of the heaviness of the other colors as compared with white. The set of colored squares was presented twice-first in a random order. then in a reverse counterbalanced order. The heaviness judgments were averaged to give one judgment per color combination per subject.

\section{RESULTS}

The data were analyzed according to a split-plot factorial analysis of variance design (Edwards, 1968). The three main factors of hue, value, and chroma were each statistically significant $(F=8.7,72.2$, 85.1, respectively; $p<.01)$. The only significant interaction was that between hue and chroma $(p<.01)$. There was no significant sex difference in assigning weight to colors.

Comparisons between means were made using Scheffé's test (Edwards, 1968). For hue, means were tested at each chroma, since there was a significant hue-chroma interaction. The only hue comparison that was significant was yellow vs. blue for chroma 8 $(p<.05)$, indicating that at this chroma yellow is judged significantly heavier than blue. No other hue comparisons were significant. Means for hues at each chroma are pletted in Figure 1.

By Scheffé's test, mean values differed significantly $(p<.01)$. In addition, $t$ tests indicated that black was judged significantly heavier than values 5,6 , and 7 , and was also significantly heavier than the modulus assigned to white $(\mathrm{p}<.01)$. The means for value are presented in Figure 2. As can be seen, apparent weight is a decreasing nonlinear function of value. The functions in Figure 2 are generally monotonic except for low chromas, for which apparent weight judgments actually become slightly less than the modulus assigned to white.

Mean chromas also differed significantly $(\mathrm{p}<.01)$. In Figure 3, it is apparent that perceived weight is an increasing function of chroma. The hue-chroma interaction is reflected in the fact that the curve for yellow (5Y) has a steeper slope than the other functions.

\section{DISCUSSION}

The present experiment demonstrates that colors do appear to have consistent weights. Hue is a relatively minor determinant. Only at the highest chroma was there a significant difference between hues, and then only between blue and yellow, with 


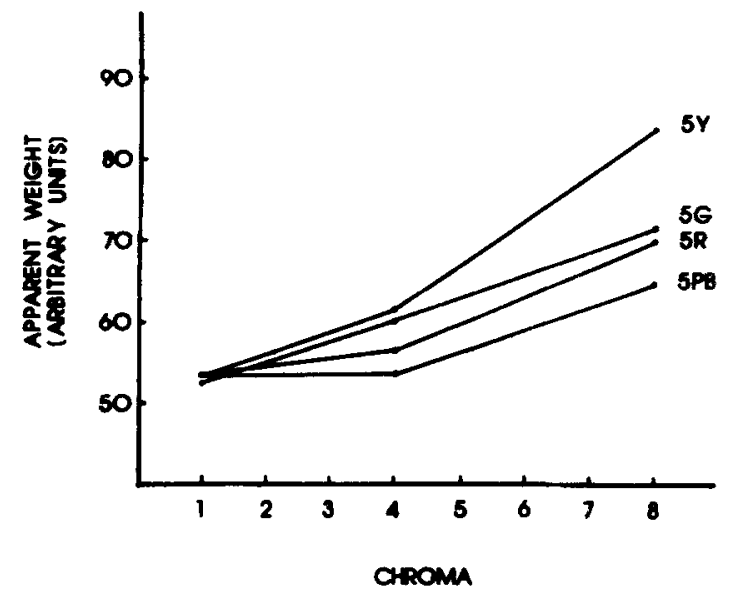

Figure 3. Apparent weight as a function of Muneell chroma. Individual function represent different hoea, a indicated. For all points, the s.e. are leas than \pm 3 mits.

yellow being heavier. Instead, value and chroma are the major determinants of color weight. Apparent weight is a decreasing function of value and an increasing function of chroma. These results support the earlier qualitative findings that "dark" colors appear heavier than "light" colors, while providing quantitative meaning to the terms dark and light. The present results do not support the conclusion of Pinkerton and Humphrey (1974), who found that hue, not brightness, determines color weight. The apparent contradiction may result from inadequate specification and control of stimulus attributes in their study.

The reason that colors appear to have different weights is not clear. Bullough (1907) first offered the reasonable suggestion that the apparent density of colors determines their apparent weight just as the actual density of objects determines their physical weight. Color weight would then be another example of a synesthetic interaction between sense modalities, in this case between vision and kinesthesis (see Marks, 1975). According to this scheme, hue, value, and chroma would interact to determine color density; then color density would determine color weight by a process of synesthesia. An appropriate test of this model would involve sensory scaling of the various attributes of color, both physical and psychological, as done for the attributes of sound by Stevens and his colleagues (Stevens, 1975).

Application of our results should be made with some caution. First, several subjects judged a few colors as lighter than white, suggesting that the relationship between apparent weight and value may be nonmonotonic under certain conditions. Secondly, the background against which the colors are seen may have some effect on apparent weight, as suggested by Monroe (1926). However, within the confines of this study, the findings are clear. Hue contributes little to the apparent weight of colors. Value and chroma are the primary determinants of color heaviness.

\section{REFERENCES}

Birren, F. Color psychology and color therapy. New York: University Books, 1961.

Burlough, E. On the apparent heaviness of colors. British Journal of Psychology, 1907, 2, 111-152.

Committe on Colorimetry (Optical Society of America). The science of color. New York: Crowell, 1953.

DECAMP, J. E. The influence of color on apparent weight: A preliminary study. Journal of Experimental Psychology, 1917, 2, 347-370.

EDWARDS, A. Experimental design in psychological research. New York: Holt, Rinehart and Winston, 1968.

IsmirarA, S. Tests for colour-blindness, 24 plates. Tokyo: Kanehara Shuppan, 1971.

Marks, L. E. On colored-hearing synesthesia: Cross-model translations of sensory dimensions. Psychological Bulletin, 1975, 82, 303-331.

MonRoE, M. The apparent weight of color and correlated phenomena. American Journal of Psychology, 1926, 36, 192-206.

PAYNe, M. C. Apparent weight as a function of color. American Journal of Psychology, 1958, 71, 725-730.

PanNe, M. C. Apparent weight as a function of hue. American Journal of Psychology, 1961, 74, 104-105.

Pepper, S. Rrinciples of art appreciation. New York: Harcourt. Brace, 1949.

Pinkerton, E., \& Humphrey, N. K. The apparent heaviness of colours. Nature, 1974, 250, 164-165.

Stevens, S. S. Psychophysics: Introduction to its perceptual, neural and social prospects. New York: Wiley, 1975.

TAYzor, C. D. Visual perception versus visual plus kinaesthetic perception in judging colored weights. Journal of General Psychology, 1930, 4, 229-246.

Trilling, M., \& Nicholas, F. Design your home for living. New York: Lippincott, 1953.

W ARDEN, C., \& FLYNN, E. The effect of color on apparent size and weight. American Journal of Psychology, 1926, 37, 398-401.

\section{NOTES}

1. For a more complete discussion of these topics, see Committee on Colorimetry (1953).

2. In the study by Pinkerton and Humphrey (1974), light from projectors passed through Wratten filters and transilluminated two circular ground-glass screens set in a matte black background. A luminous slit connected the colored circles. Subjects viewed the stimulus configuration in darkness and adjusted a luminous fulcrum under the slit to "balance" the two colored circles.

3. Munsell Color, 2441 N. Calvert Street, Baltimore, Maryland.

4. Due to pigment limitations, the Munsell color solid is asymmetrical. At high and low values, not all chromas can be reproduced across hues.

(Received for publication April 25, 1975; revision accepted September 23, 1975.) 